\title{
CONCERNING CONTINUOUS IMAGES OF RIM-METRIZABLE CONTINUA
}

\author{
H. MURAT TUNCALI
}

(Communicated by James E. West)

\begin{abstract}
Mardesic (1962) proved that if $X$ is a continuous, Hausdorff, infinite image of a compact ordered space $K$ under a light mapping in the sense of ordering, then $\omega(X)=\omega(K)$. He also proved (1967) that a continuous, Hausdorff image of a compact ordered space is rim-metrizable. Treybig (1964) proved that the product of two infinite nonmetrizable compact Hausdorff spaces cannot be a continuous image of a compact ordered space.

We prove some analogues of these results for continuous Hausdorff images of rim-metrizable spaces.
\end{abstract}

A topological space $X$ is said to be rim-metrizable if $X$ admits a basis of open sets whose boundaries are metrizable. A continuum is a compact connected Hausdorff space. Throughout the paper all spaces are assumed to be Hausdorff and all mappings are continuous.

A compact ordered space admits a basis of open sets with at most two-point boundaries. Therefore, every compact ordered space is rim-metrizable. Since 1960 there has been a lot of work done on characterizing continuous Hausdorff images of compact ordered spaces. It is natural to ask whether these results can be generalized to continuous Hausdorff images of compact rim-metrizable spaces. Some of the results that have been obtained for continuous images of compact ordered spaces are the following:

(1) In 1962 Mardešić [4] proved that if $X$ is a continuous Hausdorff image of a compact ordered infinite space $K$ under a light mapping in the sense of ordering, then the weight of $X$ equals the weight of $K$.

(2) In 1967 Mardešić [5] also proved that a continuous Hausdorff image of a compact ordered space is rim-metrizable.

(3) In 1964 Treybig [6] proved that if the product of two infinite compact spaces $X$ and $Y$ is a continuous image of a compact ordered space, then $X$ and $Y$ must be metrizable.

Received by the editors October 11, 1988 and, in revised form, August 1, 1990; the results of this paper were presented at the Spring Topology Conference, Gainesville, Florida, April 7-9, 1988.

1980 Mathematics Subject Classification (1985 Revision). Primary 54F25, 54C50; Secondary $54 \mathrm{E} 35$.

The contents of this paper constitutes a part of the author's Ph.D. thesis written under the supervision of Professor E. D. Tymchatyn. Financial support was provided by the University of Saskatchewan Graduate Scholarship. 
In this paper we prove some analogues of the above results for continuous images of rim-metrizable spaces.

Every 0-dimensional space is rim-metrizable and every compact space is a continuous image of some 0 -dimensional compact space. Thus, in order to obtain generalizations or analogues of the above results it is necessary to assume some additional conditions on the domain.

Let $L$ denote the long line and $C$ denote the Cantor ternary set. Let $X$ be the subspace of the product space $[0,1] \times L$ such that

$$
X=[0,1] \times\{0\} \cup C \times L .
$$

Then $X$ is a rim-metrizable continuum and there exists a mapping of $X$ onto $[0,1] \times L$. But $[0,1] \times L$ is not rim-metrizable and $L$ is not metrizable. This example shows that results (2) and (3) of Mardešić and Treybig, respectively cannot be generalized to continuous images of rim-metrizable spaces.

We shall prove the following analogues of (1) and (3).

Theorem 1.2. Let $X$ be a rim-metrizable continuum and let $Y$ be a continuous image of $X$ under a light mapping. Then the weight of $X$ equals the weight of $Y$.

Theorem 2.1. Let $X$ be a rim-metrizable compact space. Let $f: X \rightarrow Y \times[0,1]$ be a mapping of $X$ onto a product of a nondegenerate compact space $Y$ and $[0,1]$. If for every $y \in Y$ there exists a continuum $K$ in $f^{-1}(\{y\} \times[0,1])$ such that $f(K)=\{y\} \times[0,1]$, then $Y$ is metrizable.

In the third section of this paper, we prove that a continuous image of a rimmetrizable continuum is rim-metrizable if the mapping is monotone or open, or if the domain is locally connected and the mapping is pseudo-confluent. The example given above shows that continuous images of rim-metrizable spaces are not necessarily rim-metrizable. The space $X$ in the example is not locally connected. Hence the following question remains open: Is the continuous image of a locally connected, rim-metrizable continuum under a light mapping rimmetrizable?

\section{Preliminaries}

Let $X$ be a topological space. The weight of $X, \mathrm{w}(X)$, is the least cardinal number $\alpha$ having the property that $X$ admits a basis with cardinality $\leq \alpha$. A family $N$ of subsets of $X$ is said to be a network for $X$, if for each open set $O \subset X$ and each $x \in O$, there exists $V \in N$ such that $x \in V \subset O$. The network weight of $X, \mathrm{nw}(X)$, is the least cardinal number $\alpha$ such that there exists a network for $X$ with cardinality $\alpha$.

We denote the closure, the boundary, and the interior of a subset of a topological space by $\mathrm{Cl}(A), \operatorname{Bd}(A)$, and $\operatorname{Int}(A)$, respectively. We denote the set of rational numbers by $Q$.

The following theorems will be used later. 
Theorem 0.1. (See [1, Theorem 3.1.19, p. 171]). Let $X$ be a compact space. Then $\mathrm{w}(X)=\mathrm{nw}(X)$.

Theorem 0.2. (See [1, Theorem 3.1.22, p. 171]). Let $f: X \rightarrow Y$ be a mapping of a compact space $X$ onto $Y$. Then $\mathrm{w}(Y) \leq \mathrm{w}(X)$.

Theorem 0.3. Let $X$ be a normal space and let $B$ be a basis of open sets for the topology on $X$. Then $X$ admits a basis $B^{\prime}$ of open $F_{\sigma}$-sets such that $\operatorname{Card}(B) \geq \operatorname{Card}\left(B^{\prime}\right)$ if $\operatorname{Card}(B)$ is infinite, and $B=B^{\prime}$ if $\operatorname{Card}(B)$ is finite.

Proof. Let $B$ be a basis of open sets for a normal space $X$. If $\operatorname{Card}(B)$ is finite, since $X$ is Hausdorff, $X$ has to be finite. It follows that each open set is an open $F_{\sigma}$-set. Hence $B$ is itself a basis of open $F_{\sigma}$-sets.

Suppose $\operatorname{Card}(B)$ is infinite. Let

$$
C=\{(U, V): U, V \in B, \mathrm{Cl}(U) \subset V\} .
$$

$\{U \in B:(U, V) \in C$ for some $V \in B\}$ is a basis for $X$ because $X$ is a regular space. For each $(U, V) \in C$, using the normality of $X$, we can assign an open $F_{\sigma}$-set $B_{U V}$ such that

$$
\mathrm{Cl}(U) \subset B_{U V} \subset V .
$$

Then $B^{\prime}=\left\{B_{U V}:(U, V) \in C\right\}$ is a basis for $X$.

If $\operatorname{Card}(B)$ is infinite, by the definition of the set $C, \operatorname{Card}(C)=\operatorname{Card}(B)$. It follows that $\operatorname{Card}\left(B^{\prime}\right) \leq \operatorname{Card}(B)$.

Let $f: X \rightarrow Y$ be a mapping of a compact space $X$ onto a space $Y$. We say $f$ is pseudo-confluent, if for every irreducible continuum $K$ in $Y$, there exists a continuum $L$ in $f^{-1}(K)$ such that $f(L)=K$. We say $f$ is light, if for every $y \in Y, f^{-1}(y)$ is totally disconnected.

\section{Preservation of Weight by light mappings}

In this section, we prove that a light mapping of a rim-metrizable nondegenerate continuum preserves the weight. The aforementioned result of Mardešić shows that the weight is preserved by light mappings (in the sense of ordering) of compact ordered spaces. Mardešić [3] also proved that a light mapping of a locally connected, compact space preserves the weight. Theorem 1.2 is closely related to this result of Mardešić and it extends the result to a nonlocally connected case. By the monotone-light factorization theorem, any mapping $f: X \rightarrow Y$ of a compact space onto a space $Y$ can be represented as a composition $f=h g$ where $g: X \rightarrow Z$ is monotone (each point inverse $g^{-1}(z)$ is connected) and $h: Z \rightarrow Y$ is light. Monotone mappings do not, in general, preserve weight. Hence, we assume that the mapping considered is light.

Lemma 1.1. Let $X$ be a continuum, and let $U \subset X$ be an open set with a metrizable boundary. Let $G$ denote the upper semi-continuous decomposition of $\mathrm{Cl}(U)$ into its components. Then the quotient space $\mathrm{Cl}(U) / G$ is a compact metric space. 
Proof. Let $R=\{C \cap \operatorname{Bd}(U): C \in G\}$. By the Boundary Bumping Theorem $C \cap \operatorname{Bd}(U) \neq \varnothing$ for every $C \in G$. Consider the space $\operatorname{Bd}(U) / R$. Then $\operatorname{Bd}(U) / R$ is a compact metric space.

Define $f: \operatorname{Bd}(U) / R \rightarrow \mathrm{Cl}(U) / G$ as follows: If $g \in \operatorname{Bd}(U) / R$, let $f(g)$ be the unique component $C$ of $\mathrm{Cl}(U)$ such that $g=C \cap \mathrm{Bd}(U)$. It easily follows that $f$ is a homeomorphism. Hence $\mathrm{Cl}(U) / G$ is a compact metric space.

Theorem 1.2. Let $X$ be a nondegenerate rim-metrizable continuum and let $Y$ be the continuous image of $X$ under a light mapping $f: X \rightarrow Y$. Then $\mathrm{w}(X)=$ $\mathrm{w}(Y)$.

Proof. First note that since $X$ is a nondegenerate continuum and $f$ is a light mapping, $Y$ must also be a nondegenerate continuum. Therefore, $\mathrm{w}(X)$ and $\mathrm{w}(Y)$ are at least countably infinite.

By Theorem $0.2 \mathrm{w}(Y) \leq \mathrm{w}(X)$, because $X$ and $Y$ are compact spaces. We will show that $\mathrm{w}(X) \leq \mathrm{w}(Y)$. It is sufficient to show that $X$ admits a network with cardinality $\leq \mathrm{w}(Y)$ because of Theorem 0.1 .

Let $\left\{U_{\alpha} \mid \alpha \in A\right\}$ be a basis of open $F_{\sigma}$-sets in $Y$ such that $\operatorname{Card}(A)=\mathrm{w}(Y)$ (Theorem 0.3). Then for each $\alpha \in A, f^{-1}\left(U_{\alpha}\right)$ is an open $F_{\sigma}$-set. There exists a sequence of closed sets $F_{\alpha i}, i=1,2, \ldots$, in $X$ such that

$$
f^{-1}\left(U_{\alpha}\right)=\bigcup_{i=1}^{\infty} F_{\alpha i}
$$

and $\operatorname{Bd}\left(F_{\alpha i}\right)$ is metrizable for all $i=1,2, \ldots$.

Let $Z_{\alpha i}=F_{\alpha i} / G_{\alpha i}$ where $G_{\alpha i}$ is the decomposition of $F_{\alpha i}$ into components as in Lemma 1.1. Then $Z_{\alpha i}$ is a compact metric space, and thus, $Z_{\alpha i}$ is second countable. For each $\alpha$ and $i$, let $B_{\alpha i}=\left\{B_{\alpha i j} \mid j=1,2, \ldots\right\}$ be a countable basis for $Z_{\alpha i}$. Define $B_{\alpha i}^{\prime}=\left\{\bigcup B_{\alpha i j} \mid j=1,2, \ldots\right\}$. Note that the collection $B_{\alpha i}^{\prime}$ is a collection of subsets of $X$ lying in $F_{\alpha i}$, and also, each $B_{\alpha i}^{\prime}$ is countable. Let

$$
B=\bigcup\left\{\bigcup_{i=1}^{\infty} B_{\alpha i}^{\prime} \mid \alpha \in A\right\}
$$

Since $\operatorname{Card}(A)$ is at least countable infinite and $B_{\alpha i}^{\prime}$ is countable, $\operatorname{Card}(B) \leq$ $\operatorname{Card}(A)=\mathrm{w}(Y)$. We will show that $B$ is a network for $X$.

Let $O$ be an open set in $X$ and $x \in O$. Let $y=f(x)$. Since $f$ is a light mapping, there exists an open set $V$ such that $x \in V \subset O$ and $\operatorname{Bd}(V) \cap$ $f^{-1}(y)=\varnothing$. Now, $f$ is a closed mapping and $\operatorname{Bd}(V)$ is a closed set, therefore, $f(\operatorname{Bd}(V))$ is closed in $Y$ and $Y-f(\operatorname{Bd}(V))$ is an open set containing the point $y$. It follows that there exists $\alpha \in A$ such that $y \in U_{\alpha} \subset Y-f(\operatorname{Bd}(V))$. Note that the component $C$ of $f^{-1}\left(U_{\alpha}\right)$ containing $x$ lies in $V$; i.e. $x \in C \subset V \subset$ $O$. By (I), there exists $F_{\alpha i}$ such that $x \in F_{\alpha i}$. Obviously, the component $C_{0}$ of $F_{\alpha i}$ containing $x$ is contained in $C$. So, we have

$$
x \in C_{0} \subset C \subset V \subset O \text {. }
$$


Since $C_{0} \subset V$ and $V \cap F_{\alpha i} \neq \varnothing, V \cap F_{\alpha i}$ is an open set in $F_{\alpha i}$ containing $C_{0}$. $V \cap F_{\alpha i}$ induces an open set in the quotient space $Z_{\alpha i}$. Then there exists $B_{\alpha i j}$ for some $j$, such that $C_{0} \in B_{\alpha i j}$ and $\bigcup B_{\alpha i j} \subset V \cap F_{\alpha i}$. It follows that

$$
x \in \bigcup B_{\alpha i j} \subset V \subset O \text {. }
$$

This proves that the collection $B$ is a network for $X$ with cardinality $\leq \mathrm{w}(Y)$. Hence by Theorem $0.1 \mathrm{w}(X) \leq \mathrm{w}(Y)$.

Remark. Theorem 1.2 is not true if $X$ is assumed to be only compact and rimmetrizable. For example, let $D^{m}$ be the Cantor cube of weight $m>\aleph_{0}$ (see Engelking [1, page 115]) and let $C$ be the Cantor Ternary set. Then there exists a mapping $f: D^{m} \rightarrow C$ of $D^{m}$ onto $C$, but $\mathrm{w}\left(D^{m}\right)>\mathrm{w}(C)$.

One might also ask the question whether the assumption of rim-metrizability of the continuum $X$ in Theorem 1.2 is essential. The following example shows that the assumption "rim-metrizability" is necessary.

Example 1.3. Let $L$ denote the long line and $[0, \Omega]$ denote the set of ordinal numbers $\alpha \leq \Omega$, where $\Omega$ is the first uncountable ordinal number.

Let $X$ be the subspace of $L \times[0,1]$ such that

$$
X=L \times\{0\} \cup\{\{\alpha\} \times[0,1]: \alpha \in[0, \Omega]\} .
$$

Let $Y$ be the quotient space $X / L \times\{0\}$. Then $Y$ is a nonmetrizable continuum. It fails to be rim-metrizable at the point $\{L \times\{0\}\} \in Y$. There exists a light mapping $f: Y \rightarrow[0,1]$ of $Y$ onto $[0,1]$ (the mapping induced by the natural projection of $L \times[0,1]$ onto $[0,1])$, but $\mathrm{w}(Y)>\mathrm{w}([0,1])$.

However, if we examine the proof of Theorem 1.2 and the conditions in the hypothesis, it is easy to see that $w(Y)$ is at least countable infinite. Hence, we have the following generalization of Theorem 1.2.

Theorem 1.4. Let $f: X \rightarrow Y$ be a light mapping of a nondegenerate continuum $X$ onto a space $Y$. If $X$ admits a basis of open sets whose boundaries have weight $\leq \mathrm{w}(Y)$, then $\mathrm{w}(X)=\mathrm{w}(Y)$.

Proof. We will follow the arguments in the proof of Theorem 1.2. First, from the proof of Lemma 1.1, it is easy to see that if $U$ is an open set in a continuum $X$, then $\mathrm{w}\left(\operatorname{Bd}(U) \geq \mathrm{w}(\mathrm{Cl}(U) / R)\right.$. This implies that each of the collections $B_{\alpha i}$, $\alpha \in A, i=1,2, \ldots$, defined in the proof of Theorem 1.2 would have cardinality $\leq \mathrm{w}(Y)$. Thus the collection $B$ of the proof would have the cardinality $\leq \mathrm{w}(Y)$ and would be a network for $X$. It follows that $\mathrm{w}(X)=\mathrm{w}(Y)$.

\section{SOME PRODUCT THEOREMS}

Theorem 2.1. Let $X$ be a rim-metrizable compact space. Let $f: X \rightarrow Y \times[0,1]$ be a mapping of $X$ onto a product of a nondegenerate, compact space $Y$ and $[0,1]$. If for every $y \in Y$ there exists a continuum $K$ in $f^{-1}(\{y\} \times[0,1])$ such that $f(K)=\{y\} \times[0,1]$, then $Y$ is metrizable. 
Proof. Let $X$ be a rim-metrizable compact space and let $f: X \rightarrow Y \times[0,1]$ be a mapping of $X$ onto $Y \times[0,1]$ as in the hypothesis of the theorem, and $Y$ be a nondegenerate compact space. We will show that $Y$ admits a countable network, and by Theorem 0.1 , it will follow that $Y$ is metrizable.

Let $\Pi_{I}$ and $\Pi_{Y}$ denote the natural projections of $Y \times[0,1]$ onto $Y$, respectively. Let $r, s \in Q \cap[0,1]$ with $r<s$. Consider $f^{-1}\left(\Pi_{I}^{-1}((r, s))\right)$. Since $(r, s)$ is an open $F_{\sigma}$-set in $[0,1], f^{-1}\left(\Pi_{I}^{-1}((r, s))\right)$ is an open $F_{\sigma}$-set in $X$. For every pair $r, s \in Q \cap[0,1]$ with $r<s$, let $\left\{F_{i}^{r s}: i=1,2, \ldots\right\}$ be a sequence of closed sets with metrizable boundaries such that

$$
f^{-1}\left(\Pi_{I}^{-1}((r, s))\right)=\bigcup_{i=1}^{\infty} F_{i}^{r s} .
$$

Since $\operatorname{Bd}\left(F_{i}^{r s}\right)$ is metrizable and compact, it is second countable and has a countable network (Theorem 0.1). Let $B_{i}^{r s}$ denote a countable network for $\operatorname{Bd}\left(F_{i}^{r s}\right)$.

Let

$$
B=\bigcup\left\{\bigcup_{i=1}^{\infty} B_{i}^{r s} \mid r, s \in Q \cap[0,1], r<s\right\}
$$

and define

$$
N=\left\{\Pi_{Y}(f(C)) \mid C \in B\right\} .
$$

Now $N$ is a countable collection because each $B_{i}^{r s}$ is countable and $B$ is a countable union of $B_{i}^{r s}$ s. We shall show that $N$ is a network for $Y$.

Let $M$ be a closed subset of $Y$ and let $y \in Y-M$. Next, consider $f^{-1}(M \times[0,1])$ and $f^{-1}(\{y\} \times[0,1])$. Then $f^{-1}(M \times[0,1])$ and $f^{-1}(\{y\} \times$ $[0,1])$ are disjoint closed sets in $X$. By the hypothesis, there exists a continuum $K$ in $f^{-1}(\{y\} \times[0,1])$ such that $f(K)=\{y\} \times[0,1]$.

Let $r, s \in Q \cap[0,1]$ with $r<s$. Then, since $f(K)=\{y\} \times[0,1]$,

$$
K \cap f^{-1}\left(\Pi_{I}^{-1}((r, s))\right) \neq \varnothing
$$

and

$$
K \cap\left(X-f^{-1}\left(\Pi_{I}^{-1}((r, s))\right) \neq \varnothing .\right.
$$

Therefore there exists $F_{i}^{r s}$ for some $i$, such that $\operatorname{Bd}\left(F_{i}^{r s}\right) \cap K \neq \varnothing$. Let $x \in$ $\operatorname{Bd}\left(F_{i}^{r s}\right) \cap K$. Then $x$ is not an element of $f^{-1}(M \times[0,1])$ because $K \cap$ $f^{-1}(M \times[0,1])=\varnothing$. Thus there exists $C \in B_{i}^{r s}$ such that $x \in C$ and $C \cap f^{-1}(M \times[0,1]) \neq \varnothing$. It follows that $\Pi_{Y}(f(C)) \in N$, and $\Pi_{Y}(f(C)) \cap M=$ $\varnothing$ and $y \in \Pi_{Y}(f(C))$ because $y=\Pi_{Y}(f(x))$. This proves that $N$ is a network for $Y$. By Theorem $0.1, Y$ is metrizable.

Theorem 2.1 remains true if the domain is a rim-countable continuum or a rim-scattered locally connected continuum without any condition on the mapping (Tuncali [7]). 
Corollary 2.2. The product of a nonmetrizable, compact space and a nondegenerate continuum is not rim-metrizable.

Proof. Let $X$ be a nonmetrizable, compact space and let $Y$ be a nondegenerate continuum. Suppose that $X \times Y$ is rim-metrizable. Let $f: Y \rightarrow[0,1]$ be a mapping of $Y$ onto [0,1]. Define $g: X \times Y \rightarrow X \times[0,1]$ by $g(x, y)=$ $(x, f(y))$. Then $g$ is continuous and for every $x \in X, g^{-1}(\{x\} \times[0,1])=$ $\{x\} \times Y$. Since $f(Y)=[0,1], g(\{x\} \times Y)=\{x\} \times[0,1]$. Thus, $g$ satisfies the hypothesis of Theorem 2.1 , and therefore, $X$ is metrizable, contrary to the hypothesis.

Corollary 2.3. Let $X$ be a rim-metrizable compact space and let $f: X \rightarrow Y \times$ $[0,1]$ be a pseudo-confluent mapping of $X$ onto the product of a nondegenerate compact space $Y$ and $[0,1]$. Then $Y$ is metrizable.

Proof. By the definition of a pseudo-confluent mapping, $f$ satisfies the hypothesis of Theorem 2.1. Therefore $Y$ is metrizable.

Corollary 2.4. Let $Z$ be a nondegenerate continuous image of a rim-metrizable compact space $X$ under a pseudo-confluent mapping. Then $Z$ does not contain a product of a nonmetrizable, nondegenerate compact space $Y$ and $[0,1]$.

Proof. Let $f: X \rightarrow Z$ be a pseudo-confluent mapping of a rim-metrizable space $X$ onto a nondegenerate space $Z$. Suppose $Z$ contains a product $Y \times[0,1]$ where $Y$ is a nondegenerate compact space.

Let $f^{\prime}=f \mid f^{-1}(Y \times[0,1])$. By the definition of a pseudo-confluent mapping, it is easy to show that $f^{\prime}: f^{-1}(Y \times[0,1]) \rightarrow Y \times[0,1]$ is a pseudo-confluent mapping. Since $X$ is rim-metrizable, $f^{-1}(Y \times[0,1])$ is also rim-metrizable. Hence, the mapping $f^{\prime}$ is a pseudo-confluent mapping of a rim-metrizable compact space $f^{-1}(Y \times[0,1])$ onto $Y \times[0,1]$. By Corollary 2.3, $Y$ is metrizable.

Corollary 2.5. If the product $X \times Y$ of two nondegenerate continua is a continuous image of a rim-metrizable continuum under a pseudo-confluent mapping, then both $X$ and $Y$ are metrizable.

Proof. Let $X \times Y$ be the product of two nondegenerate continua and let $f: Z \rightarrow$ $X \times Y$ be a pseudo-confluent mapping of a rim-metrizable continuum $Z$ onto $X \times Y$.

Let $g: X \times Y \rightarrow X \times[0,1]$ be a mapping defined as in the proof of Corollary 2.2. Define $h: Z \rightarrow X \times[0,1]$ as the composition of $g$ and $f$. It is obvious that $h$ is a continuous mapping. We will show that $h$ satisfies the hypothesis of Theorem 2.1. Let $x \in X$. Then $g^{-1}(\{x\} \times[0,1])=\{x\} \times Y$. Let $K$ be an irreducible continuum contained in $\{x\} \times Y$ between points $p \in g^{-1}(x, 0)$ and $q \in g^{-1}(x, 1)$. Obviously, $g(K)=\{x\} \times[0,1]$. Since $f$ is a pseudo-confluent mapping, there exists a continuum $C$ in $f^{-1}(K)$ such that $f(C)=K$. It follows that $h(C)=g f(C)=\{x\} \times[0,1]$. This proves that $h$ satisfies the hypothesis of Theorem 2.1. It follows that $X$ is metrizable. Similarly, $Y$ is also metrizable. 
Corollary 2.6. Let $X$ and $Y$ be compact spaces. Suppose that $X \times Y$ is rimmetrizable. If both $X$ and $Y$ are not metrizable, then $X$ and $Y$ must be 0-dimensional.

Proof. Suppose that $X \times Y$ has dimension $>0$. We may assume that $X$ is not 0-dimensional without loss of generality. Let $K$ be a nondegenerate component of $X$. Then $K \times Y \subset X \times Y$, and since $X \times Y$ is rim-metrizable, so is $K \times Y$. But this contradicts Corollary 2.2.

Corollary 2.7. Let $X$ and $Y$ be compact spaces. Suppose that $X \times Y$ is rimmetrizable. If $X$ is nonmetrizable and has dimension $>0$, then $Y$ is metrizable and has dimension 0 , and $X$ is rim-metrizable.

Proof. The rim-metrizability of $X$ follows from the fact that $X \times\{y\}$ is a subspace of $X \times Y$, for some $y \in Y$. The fact that $Y$ is 0 -dimensional follows from an argument similar to that in the proof of Corollary 2.6.

In order to see that $Y$ is metrizable, let $K$ be a nondegenerate component of $X$. Since $K \times Y$ is rim-metrizable, $Y$ is metrizable by Theorem 2.1.

Theorem 2.8. Let $X$ and $Y$ be compact spaces. Then $X \times Y$ is rim-metrizable if and only if one of the following holds:

(a) Both $X$ and $Y$ are metrizable.

(b) Both $X$ and $Y$ are 0-dimensional.

(c) $X$ is rim-metrizable, and $Y$ is metrizable and has dimension 0 or vice versa.

Proof. Suppose that $X \times Y$ is rim-metrizable. If $X$ and $Y$ are not metrizable then Corollary 2.6 implies (b). If $X$ (or $Y$ ) is not metrizable and has dimension $>0$ then Corollary 2.7 implies (c).

Conversely, it is obvous that (a) implies the rim-metrizability of $X \times Y$. Assume (b). If $X$ and $Y$ are 0-dimensional compact spaces, then consider the basis

$$
B=\{U \times V \mid U \subset X, V \subset Y, U, V \text { are open, and } \operatorname{Bd}(U)=\varnothing, \operatorname{Bd}(V)=\varnothing\}
$$

for the product topology of $X \times Y$. Then for every $U \times V \in B, \operatorname{Bd}(U \times V)=\varnothing$. It follows that $X \times Y$ is rim-metrizable.

Assume (c). In this case, choose a basis as the basis $B$ above except that one of the open sets would have a metrizable boundary and the other one would have an empty boundary. Then $\operatorname{Bd}(U \times V)=\operatorname{Bd}(U) \times V$ or $U \times \operatorname{Bd}(V)$. It will follow that $\operatorname{Bd}(U \times V)$ is metrizable for all $U \times V \in B$. Thus $X \times Y$ is rim-metrizable.

\section{RIM-METRIZABILITY OF IMAGES}

Lemma 3.1. If $X$ is a compact space such that any distinct points $p, q \in X$ can be separated by a closed metrizable subset of $X$, then $X$ is rim-metrizable.

Proof. Let $x \in X$ and let $U$ be an open set containing $x$. Then $\{x\}$ and $X-U$ are disjoint closed sets. For every $y \in X-U$ let $S_{y}$ be a metrizable 
closed set which separates $x$ and $y$. Let $P_{y}$ and $Q_{y}$ be disjoint open sets such that $X-S_{y}=P_{y} \cup Q_{y}, x \in P_{y}$ and $y \in Q_{y}$. Moreover, $\operatorname{Bd}\left(P_{y}\right) \subset S_{y}$ and $\operatorname{Bd}\left(Q_{y}\right) \subset S_{y}$. Then $X-U \subset \bigcup\left\{Q_{y}: y \in X-U\right\}$. Since $X-U$ is closed in $X$, hence, compact, there exist $y_{1}, y_{2}, \ldots, y_{n}$ such that $X-U \subset Q_{y_{1}} \cup \cdots \cup Q_{y_{n}}$. Let $V=X-\mathrm{Cl}\left(\bigcup_{i=1}^{n} Q_{y_{i}}\right)$. Then $V$ is an open set such that $x \in V \subset U$. It is easy to see that $\operatorname{Bd}(V)$ is metrizable. This proves that $X$ is rim-metrizable.

Theorem 3.2. Let $X$ be a rim-metrizable continuum and let $f: X \rightarrow Y$ be a monotone mapping of $X$ onto $Y$. Then $Y$ is rim-metrizable.

Proof. Because $Y$ is compact, in order to show that $Y$ is rim-metrizable, by Lemma 3.1 it suffices to show that any pair of points $p$ and $q$ in $Y$ can be separated by a closed metrizable set in $Y$.

Let $p, q \in Y$ with $p \neq q$. Then $f^{-1}(p)$ and $f^{-1}(q)$ are disjoint, closed, connected subsets of $X$. Since $X$ is rim-metrizable, there exists a closed metrizable set $S$ in $X$ which separates $f^{-1}(p)$ and $f^{-1}(q)$ in $X$; i.e. $X-S=U \cup V$, where $U$ and $V$ are disjoint open sets, and $f^{-1}(p) \subset U$ and $f^{-1}(q) \subset V$. We shall show that $f(S)$ is a metrizable closed set which separates $p$ and $q$ in $Y$.

It is obvious that $f(S)$ is closed and metrizable because $X$ and $Y$ are compact spaces and $S$ is closed in $X$, and $f$ is a closed mapping. Suppose that $f(S)$ does not separate $p$ and $q$ in $Y$. Then $X-f^{-1}(f(S)) \subset U \cup V$ is separated in $X$ and there exists a quasi-component $C$ of $Y-f(S)$ such that both $f(U)$ and $f(V)$ intersect $C$. It follows that there exists a point $y \in Y-f(S)$ such that $f^{-1}(y) \cap U \neq \varnothing$ and $f^{-1}(y) \cap V \neq \varnothing$ (Whyburn [8, Theorem 1.3, p. 137]). But $f^{-1}(y)$ is a continuum. Therefore $f^{-1}(y) \cap S \neq \varnothing$. This implies that $y \in f(S)$ which is a contradiction. This proves the theorem.

Corollary 3.3. Let $f: X \rightarrow[0,1]$ be a mapping of a rim-metrizable continuum $X$ onto $[0,1]$. Then the quotient space which is obtained as a result of the monotone-light decomposition of $f$ is metrizable.

Proof. Theorem 3.2 and Theorem 1.2 imply the result.

Theorem 3.4. Let $X$ be a rim-metrizable continuum and let $f: X \rightarrow Y$ be an open mapping of $X$ onto a space $Y$. Then $Y$ is rim-metrizable.

Proof. Let $y \in Y$ and let $N$ be an open neighborhood of $y$ in $Y$. Then $f^{-1}(y) \subset f^{-1}(N)$ where $f^{-1}(y)$ is compact and $f^{-1}(N)$ is open. Since $X$ is rim-metrizable, there exists an open set $U$ with a metrizable boundary such that $f^{-1}(y) \subset U \subset \mathrm{Cl}(U) \subset f^{-1}(N)$. Since $f$ is an open mapping, $\operatorname{Bd}(f(U)) \subset$ $f(\operatorname{Bd}(U))$ (Whyburn [8, Theorem 7.3, p. 147]). It follows that $\operatorname{Bd}(f(U))$ is metrizable because $f(\operatorname{Bd}(U))$ is metrizable. Hence one can find a basis of open sets with metrizable boundaries for the topology of $Y$, and $Y$ is rim-metrizable.

Theorem 3.5. Let $X$ be a rim-metrizable continuum and let $f: X \rightarrow Y$ be a mapping of $X$ onto a locally connected continuum $Y$. If $f$ is pseudoconfluent then $Y$ is rim-metrizable. 
Proof. Let $p, q \in Y$ with $p \neq q$. Then $f^{-1}(p)$ and $f^{-1}(q)$ are disjoint closed subsets of $X$. Since $X$ is rim-metrizable, there exists a closed metrizable set $S$ in $X$ such that $S$ separates $f^{-1}(p)$ from $f^{-1}(q)$ in $X$. We shall show that $f(S)$ separates $p$ from $q$ in $Y$.

Suppose that $f(S)$ does not separate $p$ from $q$ in $Y$. Since $Y$ is locally connected, there exists a connected open set $C$ in $Y$ such that $p, q \in C$ and $\mathrm{Cl}(C) \cap f(S) \neq \varnothing$, (Kuratowski [2, Theorem 1, p. 238]). Let $K$ be an irreducible continuum in $\operatorname{Cl}(C)$ between $p$ and $q$. Since $f$ is pseudoconfluent, there exists a componet $L$ of $f^{-1}(K)$ such that $f(L)=K$. Since $p, q \in K$, $L \cap f^{-1}(p) \neq \varnothing$ and $L \cap f^{-1}(q) \neq \varnothing$. Thus $L \cap S \neq \varnothing$. But $f(L) \cap f(S)=\varnothing$ which is a contradiction. Hence $f(S)$ separates $p$ from $q$ in $Y$. Since $S$ is a closed metrizable subset of a compact space $X, f(S)$ is metrizable. By Lemma 3.1, $Y$ is rim-metrizable.

\section{ACKNOWLEDGMENT}

The author expresses his gratitude to Professor E. D. Tymchatyn and Professor W. Bula for their advice and discussions.

\section{REFERENCES}

1. R. Engelking, General topology, PWN, Warszawa, 1977.

2. K. Kuratowski, Topology, Vol. II, Academic Press, New York, 1968.

3. S. Mardešić, Locally connected, ordered and chainable continua, Rad Jugoslav. Akad. Znan. Umjet. 319 (1960), 161-166.

4. _ Continuous images of ordered compacta, the Suslin property and Diadic compacta, Glas. Math. Ser. III 17 (1962), 3-25.

5. __ Images of ordered compacta are locally peripherally metric, Pacific, J. Math. 23 (1967), 557-568.

6. L. B. Treybig, Concerning continuous images of compact ordered spaces, Proc. Amer. Math Soc. 15 (1964), 866-871.

7. H. M. Tuncali, Analogues of Treybig's product theorem, Proc. Amer. Math. Soc. 108 (1990), $855-858$.

8. G. T. Whyburn, Analytic topology, Amer. Math. Soc. Colloq. Publ., vol. 27, Amer. Math. Soc., Providence, RI, 1942.

Department of Mathematics, University of Saskatchewan, Saskatoon, Saskatchewan, CANADA S7N OW0 